\title{
Realistic Simulation of IEEE 802.11p Channel in Mobile Vehicle to Vehicle Communication
}

\author{
Tarikul Islam, Yongchang Hu, Dr. Ertan Onur \\ Faculty of EEMCS \\ Delft University of Technology \\ Delft, The Netherlands \\ e-mail: tarekdelft@gmail.com,\{y.hu-1,e.onur\}@tudelft.nl
}

\author{
Dr. Bert Boltjes ${ }^{1}$, Dr. J.F.C.M de Jongh ${ }^{2}$ \\ ${ }^{1}$ Modeling Simulation \& Gaming, ${ }^{2}$ Network Technology \\ Netherlands Organization for Applied Scientific Research \\ Delft, The Netherlands \\ e-mail: \{bert.boltjes, jan.dejongh\}@tno.nl
}

\begin{abstract}
Intelligent Transportation Systems (ITS) is becoming an important paradigm, because of its ability to enhance safety and to mitigate congestion on road traffic scenarios. Realizing the fact that data collection scheme from in-situ test beds for large number of vehicles is always expensive and time consuming. Before being employed in large scale, such safety critical system should be tested narrowing down the gap between real circumstances and analytical models in a simulation platform. It is evident that underlying radio wave propagation models can comprise the validity of large scale vehicular network simulation results. Vehicle-to-Vehicle (V2V) channels have higher dynamics due to rapidly varying topologies and environments which have significant impact on performance study of upper layer protocols and applications. In spite of the fact that few measurement based empirical channel models are present in the literature, they are not tested for large scale vehicular networks. In this study, we simulate suburban scenarios with hundreds of IEEE802.11p nodes in the OPNET simulation environment with more realistic channel models. The standard OPNET propagation model was replaced by Nakagami-m fading channel. For the sake of modeling, changing relative velocity attribute and separation distance, power spectrum and fading parameter- $m$ were defined as function of velocity and separation distance respectively. Then statistics were collected to evaluate performance of physical and higher layers. Primarily we have found all the vehicles within the standard requirement for Dedicated Short Range Communications (DSRC) range of 1 kilometer may not receive packets, which was also found in several earlier publications.
\end{abstract}

Keywords-ITS, VANET, IEEE802.11p, Nakagami-m fading.

\section{INTRODUCTION}

In recent years, required development for wireless vehicular network is being realized as it can contribute effectively to look-ahead vehicle collision avoidance, alleviate congestion, lane change, and construction site warning. An IEEE task group has approved the IEEE $802.11 \mathrm{p}$ standard to enhance 802.11 capabilities to support ITS. The proposed standard will be used as a base element of DSRC for short and medium range wireless communications in vehicular scenarios. To enhance public safety, in 1999 United States Federal Communication Commission (FCC) allocated $75 \mathrm{MHz}$ bandwidth of DSRC at $5.9 \mathrm{GHz}$ frequency for Vehicle to Vehicle (V2V) and Vehicle to Infrastructure (V2I) communications [1]. For harmonized use, in the year of 2008 Commission of European Communities (CEC) assigned 5875 $\mathrm{MHz}$ to $5905 \mathrm{MHz}$ frequency spectrum for Intelligent Transportation Systems [2]. However, foreseeable real time applications demand reliable channel models tested with large number of vehicles before being implemented in road traffic scenarios.

To a great extend, the accuracy of large scale simulation of vehicle to vehicle (V2V) communication depends upon underlying propagation channel as $\mathrm{V} 2 \mathrm{~V}$ channels have strong influence over coverage, reliability, and real time capabilities [3]. Simulation of large scale V2V communications with appropriate channel models is still under investigation. The research community has invested significant effort to derive realistic channel models for specific vehicular scenarios. Though empirical models for $\mathrm{V} 2 \mathrm{~V}$ communication were studied in several papers, pertinence of those models for large number of vehicles still remained under question as most of the simulation studies were based on free space or two ray propagation model. The impact of buildings and distance attenuation on received signal was presented in [5] without stating the effects of multipath propagation. Position based message forwarding strategy was used in [6], where it was assumed that each vehicle will periodically disseminate status information. However, a DSRC station uses demand beacons that need not be periodically repeated [7]. For the sake of achieving faster simulation, hybrid method was used for large number of vehicles in [10]. But both in [6] and [10], Nakagami fading model was implemented for fixed fading parameter- $m$ and constant average velocity. Since, V2V propagation environment changes with vehicle mobility, all the channels will not necessarily suffer same fading. In [9], the SHIFT simulator was used for large number of IEEE 802.11a radios to evaluate V2V safety messaging. For simulations of Medium Access Control and higher layers an error model approach was presented in [11] and validated using Wireless Access Radio Protocol 2 (WARP2) simulator. A two ray interference model was presented in [12] to replace simplified two ray model. Computationally inexpensive radio shadowing model was studied in [13]. All these contributions were again based on free space or two ray propagation model that does not consider multipath propagation and based on optimistic assumption of real life scenarios. Overall, there is a research gap between wireless channel models used in large scale simulations and what the channels actually are in vehicle to vehicle communication.

Following the stated absence, we tried to bring out more realistic channel model for large scale simulation of $802.11 \mathrm{p}$ 
nodes in OPNET. Our major effort was to achieve realistic simulation results for V2V communication. Furthermore, we have found that using overly optimistic channel models can substantially alter the results which are unusable for implementation in real life safety critical systems.

Like most of the existing network simulators OPNET does not have IEEE $802.11 \mathrm{p}$ communication and stochastic channel models (e.g. Nakagami). The Netherlands Organization for Applied Scientific Research (TNO) developed IEEE 802.11p node model for performance evaluation of ITS which was used in this simulation study. Moreover, required adjustments to the node model were made to represent IEEE amendment for DSRC. A Nakagami- $m$ channel model was programmed to replace OPNET default free space propagation model. The Nakagami- $m$ parameter was varied in relation to the distance between $\mathrm{Tx}$ and $\mathrm{Rx}$ to address varying multipath anomalies. Since, both $\mathrm{Tx}$ and $\mathrm{Rx}$ are in motion the autocorrelation function presented in [8] and [19] was used in the simulation model to address varying velocity attributes. Dual Slope Piecewise Linear (DSPL) model with empirical parameter values, given in [1], was used for defining realistic large scale path loss on suburban roads. Hundreds of vehicles with specific trajectory settings were simulated to retrieve statistics. The results were then compared with other propagation models (i.e., Free Space, Two Ray, and DSPL).The comparison showed considerable difference between overly simplified channel models and the realistic simulator results.

The paper is organized as follows, in Section II the $802.11 \mathrm{p}$ node model is presented, large scale and small scale fading are described in II. $A$ and II. $B$, Section III outlines simulator setup, results are presented in the sub-sections of III. $A, B, C$, and $D$, finally, section IV concludes this paper outlining results.

\section{IEEE 802.11P CHANNEL MODELS}

The used 802.11p node model is depicted in Fig.1 which shows layered architecture of the model. In this paper, our discussion will be limited to physical layer of the model. Detail about the $802.11 \mathrm{p}$ standard will not be discussed here. An overview of DSRC specifications can be found in [7]. However, the used model parameter settings will be discussed in simulation section.

\section{A. Dual Slope Piecewise Linear (DSPL) Pathloss Model}

The DSPL model is a special case of piece wise linear model for large scale fading. Empirical studies depict, the dual slope pathloss model fits measurements more accurately. This piecewise model can be charaterized with a pathloss exponent $\gamma_{l}$ and a standard deviation $\sigma_{l}$ within a critical distance $d_{c}$. If waves travel more than $d_{c}$, their power decays with another pathloss exponent $\gamma_{2}$ with a standard deviation $\sigma_{2}$. The received power $P$ can be expressed as,

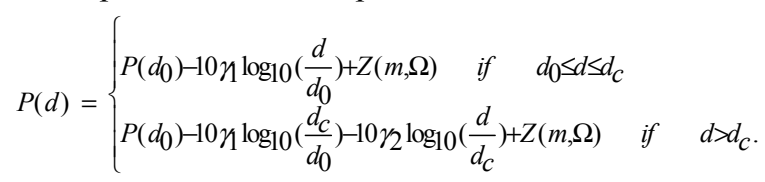

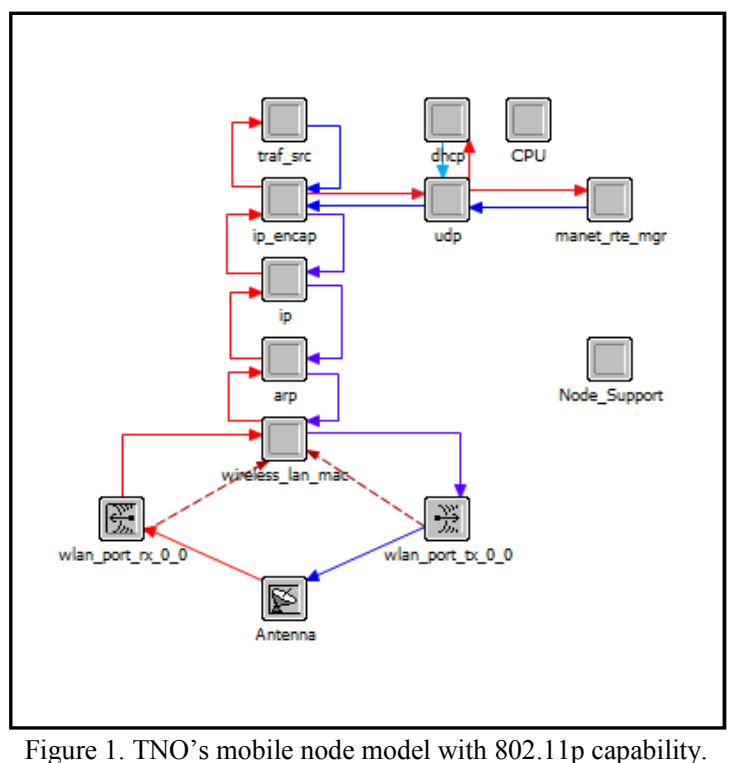

Where, $d_{0}$ is a reference distance, $m$ is the Nakagami fading parameter and $\Omega$ is the shape parameter. Since, IEEE $802.11 \mathrm{p}$ is standardized for short range communication, it is logical that V2V communication will be dominated by a strong Line of Sight (LOS) component with minor impact of shadowing. As a result, random variable $Z$ should not be Gaussian distributed, rather has statistics determined by multipath fading [1], [3]. This propagation anomaly can be stochastically modeled using Rician or Rayleigh fading. However, Nakagami fading was used in this study which is more generalized form of modeling small scale fading and can be transformed into Rayleigh or Rician fading. Dual slope pathloss exponents and critical distance values used in the simulation are presented in Table. I [1]. In the following section small scale fading is discussed.

\section{B. Nakagami Fading}

The Nakagami- $m$ distribution can model signal fading condition that ranges from severe to moderate, to light fading or no fading [14]. Empirical measurements reveal that this distribution fits better for vehicle to vehicle communication. Nakagami has presented in [15] that signal amplitude fading can be expressed as Probability Density Function (PDF),

$$
f_{R}(R)=\frac{2 m^{m} R^{2 m-1}}{\Gamma(m) \Omega^{m}} e^{-(m / \Omega) R^{2}},
$$

where, channel amplitude $R \geq 0, \Omega=E\left[R^{2}\right]=\overline{R^{2}}$ is the average fading power, $E[\cdot]$ is the expectation operator, $\Gamma(\cdot)$ is the Gamma function.

TABLE I. DUAL Slope Path Loss Parameters.

\begin{tabular}{|c|c|}
\hline Parameter & Value \\
\hline$\gamma_{1}$ & 2.1 \\
\hline$\gamma_{2}$ & 3.8 \\
\hline$d_{c}$ & 100 \\
\hline
\end{tabular}


The fading parameter $m$ which determines severity of fading can be expressed as,

$$
m=\frac{\left(\overline{R^{2}}\right)^{2}}{\left(R^{2}-\overline{R^{2}}\right)^{2}} \geq \frac{1}{2} .
$$

Cheng presented two datasets from suburban empirical measurements in [1] where he estimated shape parameter for different distance bins. To express Nakagami- $m$ parameter in relation to separation distance between $\mathrm{Tx}$ and $\mathrm{Rx}$ we applied linear regression on data set 1 of [1]. We have observed while applying linear regression that data set 1 fits better compared to the other dataset. The best fitted relation can be derived as,

$$
m=-0.69 \ln (d)+4.929 \text {. }
$$

We employed this relation during simulation to let the shape parameter vary with separation distance. Equation (3) and used data set is plotted in Fig. 2 where we can see depending on distance, the value of $m$ parameter can fluctuate within the range of 0.16 to 5.8. However, calculating fading power for the value of $m$ below 0.5 is computationally very expensive as a result any value smaller than 0.5 was considered as 0.5 .

For the sake of representing transmitter and receiver velocity omni-directional antennas and isotropic scattering condition around $\mathrm{Tx}$ and $\mathrm{Rx}$ were assumed. The normalized time autocorrelation function of received complex envelope of two mobile vehicles can be expressed as [8], [19],

$$
\Re_{g g}(\tau)=J_{0}\left(2 \pi f_{D} \tau\right) J_{0}\left(2 \pi a f_{D} \tau\right) \quad \text { where } \quad a=\frac{v_{2}}{v_{1}} .
$$

Above, $J_{0}$ is the zero order Bessel function of first kind, $f_{D}$ is the maximum Doppler frequency shift, and $a$ is the ratio of two maximum Doppler frequencies or Tx (v1) and Rx (v2) speeds which has value in between $0 \leq a \leq 1$, assuming $v 1>v 2$.

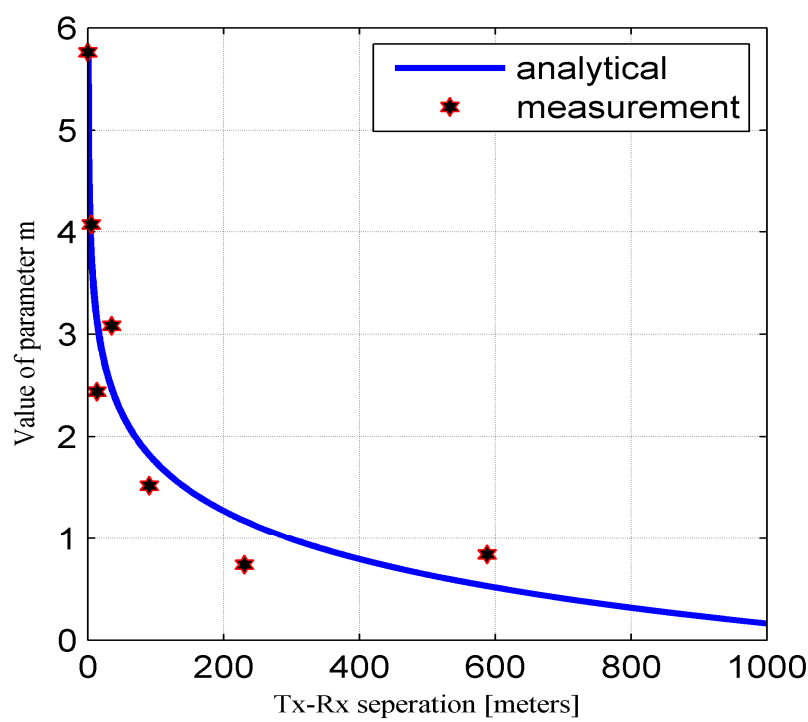

Figure 2. Relation of Nakagami- $m$ parameter with distance.
If $v 1<v 2$, then $a=\frac{v 1}{v 2}$, to keep the value of $a$ within the given range. Since, fading power spectrum is calculated by taking Fast Fourier Transform (FFT) of the autocorrelation function, this limit is applied to maintain the power spectrum so that the fading envelope would not blow up. It should be noted that for uniformly distributed random phase between 0 and $2 \pi$, Doppler frequency experienced by each path is the sum of the motion induced by motion of $\mathrm{Tx}$ and $\mathrm{Rx}$ individually. It is also noticeable in (4) that the autocorrelation function is a product of two Bessel functions instead of one Bessel function in conventional cellular communication. Equations (3) and (4) were used in the simulator described in the following section.

\section{REALISTIC IEEE802.11P SIMULATION}

The location of the simulation scenario was taken as Hoofdorp which is a typical suburban area near Amsterdam, The Netherlands. The relevant attributes of the simulator is presented in Table. II. We simulated a scenario where 169 TNO $802.11 \mathrm{p}$ nodes were distributed over total of 15 kilometers $(\mathrm{km})$ length of road. Nevertheless, depending on the length and width of the roads, the density of vehicles were 10 to 20 cars per square kilometers. The simulation was run for five minutes. Fig. 3 shows the initial distribution of the vehicles. The red cars were moving in the opposite direction as light blue cars on the same road. No lanes were considered though the roads were wide enough to overtake or cross one another. Each and every vehicle were assigned specific trajectory to follow a predefined path with varying velocity between 20-45 kilometers per hour. To keep the figure clutter free the trajectories are not shown in the plot. Cars were coming or moving away in burst due to lower speed limit and redundancy of traffic lights in suburban scenario. It is apparent that vehicle density depends on the time of the day. However, in our scenario the average value of vehicle distribution was considered. The same scenario was used to run the simulator with other three different channel models like Free Space, Two Ray, and already discussed DSPL model for validation. OPNET has 13 different pipeline stages to establish communication between a transmitter and multiple receivers. However, our major concern was how much power was available to the receivers. In Fig. 4, Rx node attributes are presented, where ragain model calculates receiver antenna gain, received power is determined by used power model, and bkgnoise is executed immediately after return of the received power stage to represent the effect of all hardware noise.

TABLE II. Simulator SetTings.

\begin{tabular}{|c|c|}
\hline Attributes & Values \\
\hline Frequency & $5.850 \mathrm{GHz}$ \\
\hline Bandwidth & $10 \mathrm{MHz}$ \\
\hline Transmit power & $23 \mathrm{dBm}$ \\
\hline Antenna gain & $0.0 \mathrm{~dB}$ \\
\hline Data rate & $6 \mathrm{Mbps}$ \\
\hline Packet format & MAC \\
\hline Modulation & BPSK \\
\hline Multiplexing & OFDM \\
\hline Antenna height & $1.51 \mathrm{~m}$ \\
\hline Node velocity & $20-45 \mathrm{Km} / \mathrm{hr}$ \\
\hline
\end{tabular}




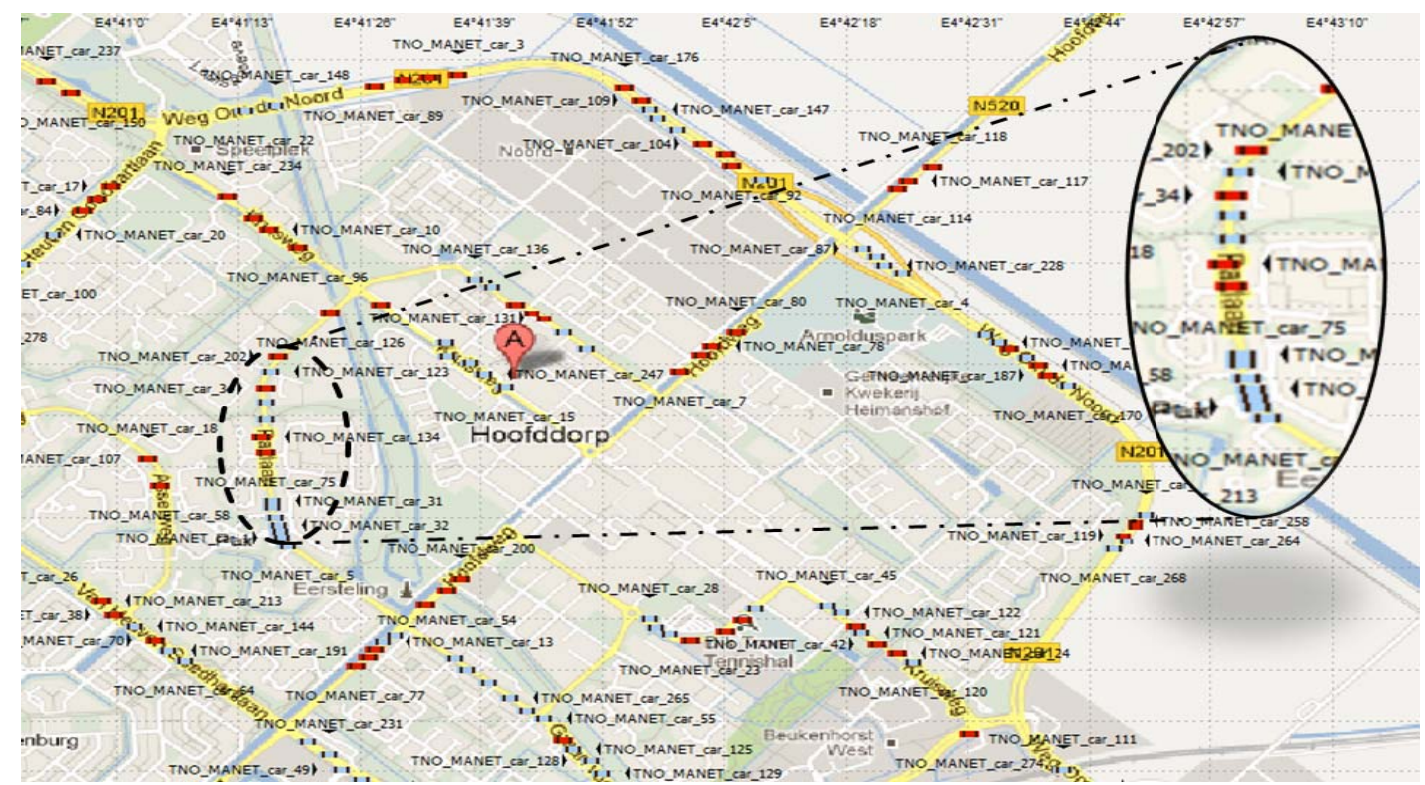

Figure 3. Suburban scenario with 169 mobile vehicles.

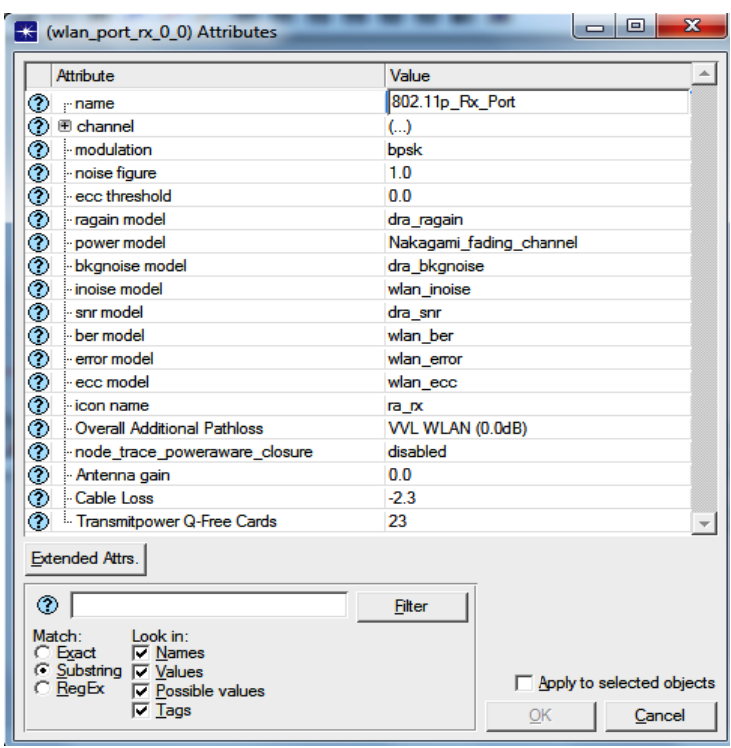

Figure 4. Pipeline stages used in the node model.

The inoise model calculates accumulated noise due to concurrent transmission during a packet reception. Signal to Interference and Noise Ratio (SINR) is estimated by snr model. Whereas, The ber model calculates Bit Error Rate (BER) on each packet segments based on previously computed effective SINR and modulation curve assigned to the receiver.

\section{A. Critical Communication Range}

To implement large scale fading the DSPL model presented in (1) was programmed in $\mathrm{C}++$ to replace OPNET standard pipeline stage power model. The simulated statistics were exported to MATLAB and reprocessed to accomplish better representation of the figures. In Fig. 5, the simulated result for large number of vehicles with empirical pathloss model is presented, where it is clearly noticeable that simulated data resembles theoretical model. The figure depicts the vehicles can come as close as 0.3 meters due to their mobility over assigned trajectory. The critical distance which is expected to be 100 meters is also observable after that signal strength falls off rapidly due to greater pathloss exponent. Furthermore, with the transmit power of $23 \mathrm{dBm}$ the maximum attainable range of communication is roughly 386 meters though the IEEE amendment for $802.11 \mathrm{p}$ specifies allowable communication range of $1 \mathrm{~km}$ for DSRC. The received power threshold was set to $-89 \mathrm{dBm}$. The noise floor of the receiver is $-104 \mathrm{dBm}$ for a system with bandwidth of $10 \mathrm{MHz}$. The difference between threshold level and noise floor can be explained by taken into account noise figure and other system parameters like signal detection performance.

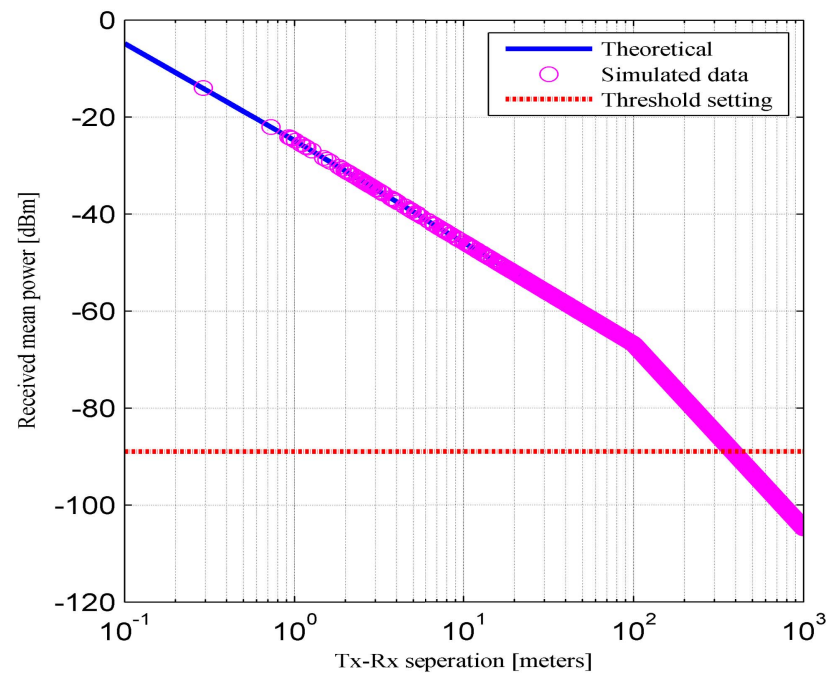

Figure 5. Simulated Dual Slope Piecewise Linear received mean power. 


\section{B. Fading and Doppler Effect}

Vehicle to vehicle communication differs from cellular network because there is no fixed based station and vehicle speed contributes Doppler shift to modulate the carrier frequency. The Doppler shift can have significant impact on signal fading. The time correlation property of the fading envelope was calculated using (4). Since, the power spectrum of the fading envelope is calculated from autocorrelation function, change in this correlation function has direct impact on signal level fluctuation of received signal as a result models velocity attributes of the Tx and Rx node. The Nakagami- $m$ fading envelope described in Section II. $B$ was applied over large scale pathloss obtained using the DSPL model. In Fig. 6, it is noticeable that with propagation distance the severity of fading increases. This is due to the fact parameter $m$ was varied with distance. Till 297 meters the $m$ parameter remains above the value of 1 . As a result, within this range nearly no fading to Rician fading occurs. However, beyond 297 meters of separation distance $m$ parameter becomes less than one. Consequently, harsher Rayleigh fading occurs beyond this distance when line of sight component of the signal is not present which is also noticeable from the figure. In the case of suburban scenario, due to low density of vehicles it is evident that line of sight component will be present in nearby distances. However, with increasing distance more multipath components will appear owing to reflections from buildings and scattering environment in the vicinity of roads. Fig. 6 explains these real life multipath propagation anomalies and shows the proposed method can represent these phenomena. Critical distance at 100 meters is visible within which signal strength fluctuates without crossing the threshold. Nevertheless, packet error rate (PER) will increase substantially beyond 100 meters for the threshold setting of $89 \mathrm{dBm}$. To compensate this problem transmit power or receiver sensitivity needs to be increased.

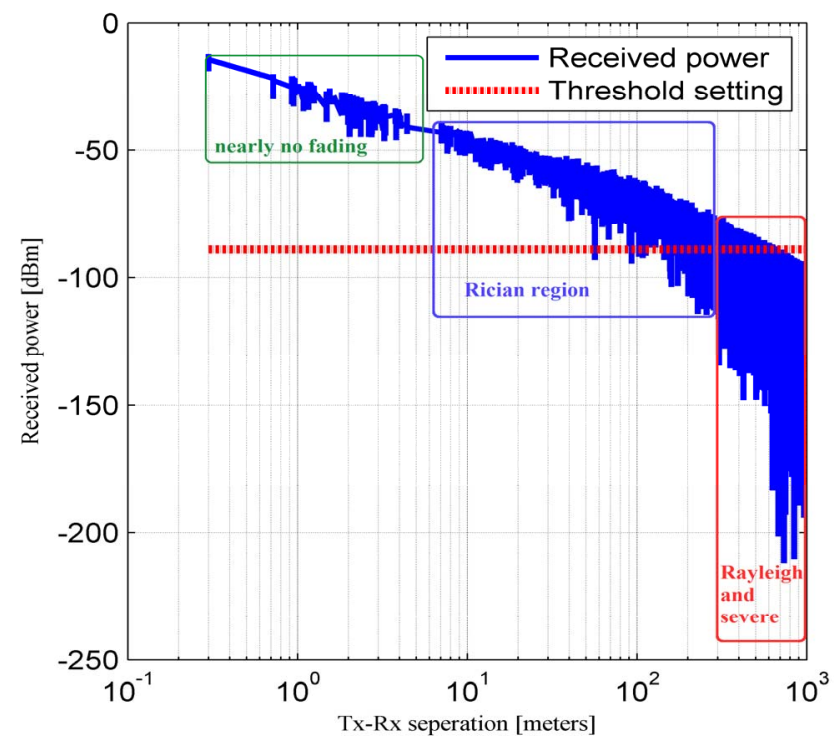

Figure 6. Simulated received power over propagation distance.

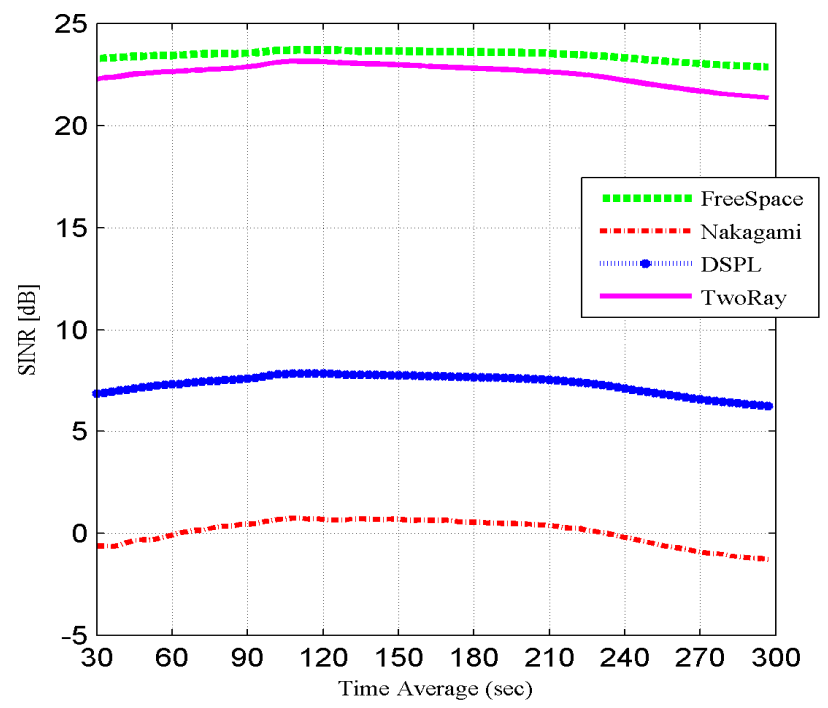

Figure 7. SINR for four different channel models.

\section{Signal to Interference and Noise Ratio (SINR)}

To address interference SINR can be defined as,

$$
S I N R=\frac{P}{N+I},
$$

where, $P$ is the received power, $N$ is the receiver's back ground noise, and $I$ is the accumulated interference noise contributed by simultaneous transmission within the range. In Fig. 7, retrieved SINR values for four different channel models simulated in the same suburban scenario are presented. The SINR values were calculated using (5) during simulation. Then time averages of samples were derived within 3 second bins to plot the figure which made it easier to interpret the result. From Fig. 7, it can be observed that there is an immense difference of about $23 \mathrm{~dB}$ between SINR retrieved using Free Space Propagation model and proposed realistic channel model. Whereas, marginal differences were obtained between Free Space propagation and Two Ray model. Nevertheless, the proposed channel model showed less discrepancy $(6 \mathrm{~dB})$ with the empirical DSPL model which validates the proposed model we have presented here. Compared to rest of the three models, Nakagami channel model showed heavily fluctuating SINR in the receiver due to presence of multipath components of the signal and varying Doppler frequency imposed by Tx-Rx velocity (not shown in Fig. 7) which is evident for V2V communication.

\section{Bit Error Rate (BER)}

As expected, Fig. 8 depicts that simulated Nakagami model attained higher BER compared with other three models. Till 50 seconds out of 1000 bits around 2 bits were misinterpreted by the receiver due to very weak SINR values. On the other hand, Free Space and Two Ray model showed identical Bit Error Rate as they returned similar Signal to Interference and Noise Ratio. Again, the realistic propagation model proposed in this study showed less difference with Dual Slope Piecewise Linear pathloss model as latter model invoked 


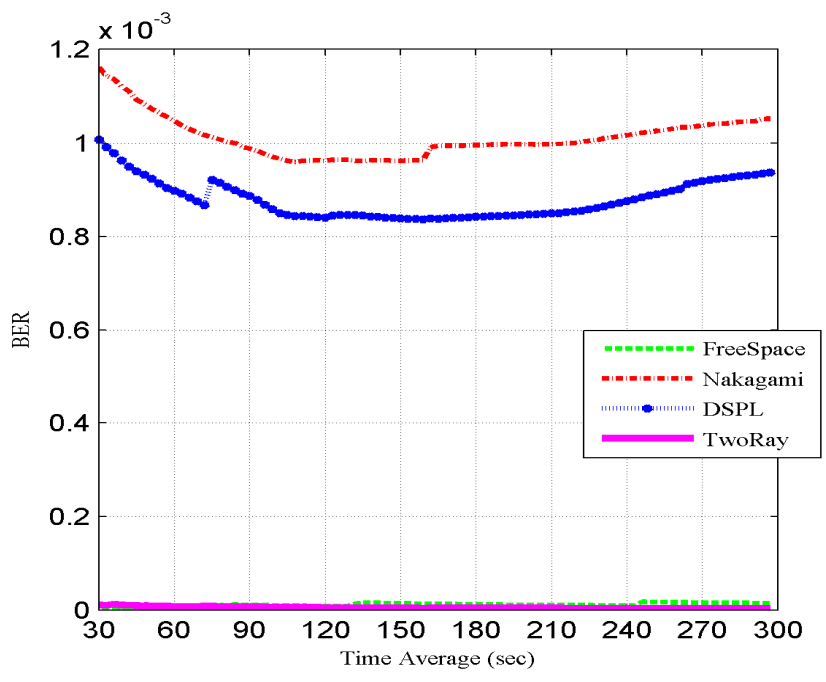

Figure 8. BER for four different channel models.

pathloss exponents derived from real life experiments.

From the consequences of Fig.7 and Fig.8, it can be deduced that depending on the complexity of the model or the used method to develop the model can greatly influence obtained simulation results.

\section{CONCLUSION}

In this study, we have presented a realistic wireless channel model for simulation of large scale vehicle to vehicle communication. Initially it was found that maximum attainable range for DSRC is around 386 meters by considering large scale signal level attenuation only. The additional techniques applied to Nakagami- $m$ fading channel for the simulation of mobile vehicle to vehicle communication demonstrated that those methods can successfully exemplify nearly no fading to Rician fading, and Rayleigh fading to more severe multipath fading. Then we compared the proposed small scale fading channel model with other counterparts. The comparison showed that overly simplified model for the mentioned use case may imply optimistic results which were unrepresentative of the original propagation anomalies. The presented model not only can be used as realistic presentation of underlying environment but also for large scale simulation and performance evaluation of higher layer protocols for ITS applications. In our future work, we will analyze Medium Access Control (MAC) layer and protocols above MAC layer for $\mathrm{V} 2 \mathrm{~V}$ communication.

\section{ACKNOWLEDGMENT}

The authors would like to thank Martijn van Noort and Jaap van den Oever of TNO for their contribution to set up suburban scenario and physical layer parameters. We would also like to appreciate the support from OPNET Technologies, Inc. for providing OPNET Modeler to TU Delft.

\section{REFERENCES}

[1] L. Cheng, B. Henty, D. Stancil, F. Bai and P. Mudalige, "Mobile vehicle-to-vehicle narrow-band channel measurement and characterization of the $5.9 \mathrm{GHz}$ dedicated short range communication (DSRC) frequency band," IEEE Journal on Selected Areas in Communications, vol. 25, no. 8, pp. 1501 -1516, 2007.
[2] "Commision decision on the harmonised use of radio spectrum in the $5875-5905 \mathrm{MHz}$ frequency band for safety-related applications of Intelligent Transport Systems (ITS).” 2008/671/EC, August 2008.

[3] G.P. Grau, D. Pusceddu, S. Rea, O. Brickley, M. Koubek, D. Pesch, "Vehicle-2-vehicle communication channel evaluation using the CVIS platform," IEEE 7th International Symposium on Communication Systems Networks and Digital Signal Processing (CSNDSP), pp.449453, 21-23 July 2010.

[4] L. Cheng, B. Henty, F. Bai, D. Stancil, "Highway and rural propagation channel modeling for vehicle-to-vehicle communications at 5.9 GHz," IEEE Antennas and Propagation Society International Symposium, 2008, pp.1-4, 5-11 July 2008

[5] F.J . Martinez, Toh Chai-Keong Toh, J.-C. Cano, C.T. Calafate, P. Manzoni, "Realistic Radio Propagation Models (RPMs) for VANET Simulations," IEEE Wireless Communications and Networking Conference, 2009, pp.1-6, 5-8 April 2009.

[6] M. Torrent-Moreno, "Inter-vehicle communications: assessing information dissemination under safety constraints," IEEE Fourth Annual Conference on Wireless on Demand Network Systems and Services, pp.59-64, 24-26 Jan. 2007.

[7] D. Jiang, L. Delgrossi, "IEEE 802.11p: Towards an International Standard for Wireless Access in Vehicular Environments," IEEE Vehicular Technology Conference, 2008, pp.2036-2040, 11-14 2008.

[8] A. S. Akki and F. Haber, "A statistical model for mobile-to-mobile land communication channel," IEEE Trans. on Veh. Technol., vol. VT-35, no. 1, Feb. 1986.

[9] Q. Xu, T. Mak, J. Ko, and R. Sengupta, "Vehicle-to-vehicle safety messaging in DSRC," Proceedings of the 1st ACM international workshop on Vehicular ad hoc networks (VANET '04), pp.19-28, 2004.

[10] M. Killat, F. Schmidt-Eisenlohr, H. Hartenstein, C. Rössel, P. Vortisch, S. Assenmacher, and F. Busch, "Enabling efficient and accurate large scale simulations of VANETs for vehicular traffic management," Proceedings of the fourth ACM international workshop on Vehicular ad hoc networks (VANET '07),pp.29-38, 2007.

[11] Y. Zang, L. Stibor, G. Orfanos, S. Guo, and H-J. Reumerman, “An error model for inter-vehicle communications in highway scenarios at $5.9 \mathrm{GHz}$," Proceedings of the 2nd ACM international workshop on Performance evaluation of wireless ad hoc, sensor, and ubiquitous networks (PE-WASUN '05),pp.49-56, 2005.

[12] C. Sommer, S. Joerer, F. Dressler, "On the applicability of Two-Ray path loss models for vehicular network simulation," IEEE Vehicular Networking Conference (VNC), pp.64-69, 14-16 Nov. 2012.

[13] C. Sommer, D. Eckhoff, R. German, F. Dressler, "A computationally inexpensive empirical model of IEEE $802.11 \mathrm{p}$ radio shadowing in urban environments," IEEE Eighth International Conference on Wireless OnDemand Network Systems and Services (WONS), pp.84-90, 26-28 Jan. 2011.

[14] N. C. Beaulieu and C. Cheng, "An Efficient Procedure for Nakagami- $m$ Fading Simulation," in Proc. of IEEE Globecom 2001, volume 6, pp.3336-3342, Nov 2001.

[15] M.D. Yacoub, M.V. Barbin, M.S.de Castro, J.E. Vargas B., "Level crossing rate of Nakagami- $m$ fading signal: field trials and validation," IEEE Electronics Letters , vol.36, no.4, pp.355-357, 2000.

[16] R.J. Punnoose, P.V. Nikitin, D. Stancil, "Efficient simulation of Ricean fading within a packet simulator," IEEE Vehicular Technology Conference, vol. 2, pp.764-767, 2000.

[17] Q.T. Zhang, "A decomposition technique for efficient generation of correlated Nakagami fading channels," IEEE Journal on Selected Areas in Communications, , vol.18, no.11, pp.2385-2392, Nov. 2000.

[18] M.I. Azam, A.U.H. Sheikh, "Error performance over frequencyselective variable Nakagami fading channel with RAKE reception," IEEE 13th International Multitopic Conference, pp.1-6, 1415 Dec. 2009

[19] C.S. Patel, G.L. Stuber, and T.G. Pratt, "Simulation of Rayleigh-Faded Mobile-to-Mobile Communication Channels," IEEE Transactions on Communications, vol.53, no.10, pp.1773, Oct. 2005. 